\title{
Noninvasive measurement of eye retraction during blinking
}

\author{
David Mas, * Begoña Domenech, Julián Espinosa, Jorge Pérez, Consuelo Hernández, and Carlos Illueca \\ Instituto de Física Aplicada a las Ciencias y Tecnologías, Universidad de Alicante, PO Box 99, 03080 Alicante, Spain \\ *Corresponding author: david.mas@ua.es
}

Received January 27, 2010; revised April 16, 2010; accepted May 2, 2010;

posted May 6, 2010 (Doc. ID 123372); published May 27, 2010

\begin{abstract}
We present a noninvasive technique for high-speed measuring of eye retraction and eyelid position during blinking. The anterior chamber of the eye is illuminated by the slit lamp of a biomicroscope and eye dynamics during a blinking sequence are captured with a high-speed camera working at 500 frames per second. Digital image processing allows quantitative analysis of cornea and eyelid positions during the closing and opening phases of the blinking process. Our method allows simultaneous measuring of corneal retraction, duration of down and up phases, total blinking duration, and average and peak speeds of the eyelids in both phases, thus providing a complete analysis of the blink's transversal motions. (c) 2010 Optical Society of America

OCIS codes: $\quad 070.2025,200.4740$.
\end{abstract}

Blinking is an essential function of the eye that helps to spread the tear film and remove foreign particles from the cornea and conjunctiva [1]. Aside from the evident eyelid activity, blinking is a complex biomechanical process. When the blinking process starts, the eye moves around $2^{\circ}$ in the inferior nasalward direction while it performs a cyclotorsional rotation. Simultaneously with these movements, the eye performs a retraction inside the orbit of $0.5-1.5 \mathrm{~mm}$ [2]. The physiological function of these movements is still not clear. It is probable that eye retraction during blinking prevents excessive pressure of the eyelid over the cornea. This pressure can provoke changes in the intraocular pressure, which could be important in fluid dynamics in the anterior chamber. Eye movements during blinking are also important because they affect the dynamics of lid-contact-lens interaction [3]. Thus, measuring such movements is of great interest for ophthalmic research.

Nowadays, scleral search coil systems provide precise eye movement measurements during blinking with a spatial resolution lower than $1 \mu \mathrm{m}$ and a temporal resolution of $1 \mathrm{~ms}$ [4]. Although this method is considered the gold standard in oculomotor research, its use is limited because it is highly invasive and uncomfortable. Technological advances have produced noninvasive image-based techniques for detecting eye movement during blinking [5]. Recently, a high-speed OCT system was presented [6] $]$. Among its capabilities, the system is able to register blinking-related eye movements at a rate of 50 frames per second (fps). The main drawback of noninvasive systems is the amount of light needed, which limits both the speed and the accuracy of the measurement. In this sense, the fastest method we found in the literature was presented by Collins et al. [3] , achieving $250 \mathrm{fps}$ and accuracy of $100 \mu \mathrm{m}$. We propose a quantitative noninvasive method that can record data up to $500 \mathrm{fps}$ with a spatial accuracy of $25 \mu \mathrm{m}$. It is based on the use of a conventional slitlamp biomicroscope and a high-speed camera. Proper image processing provides accurate measurement of eye retraction within the orbit. Furthermore, our system is the first proposal, to the best of our knowledge, that permits simultaneous measurement of eye retraction and eyelid displacement.
Nine subjects were asked to participate in the study. We adhered to the tenets of the Declaration of Helsinki. Each subject was asked to blink naturally during $6 \mathrm{~s}$ while a video sequence of the eye was captured. Three sequences per subject were registered. Since the aim of the measurement was to register fine eye movements, all residual movements had to be minimized, so a reinforced chinrest was used to hold and stabilize the head of the observed subjects. The experimental system was comprised of a Haag-Streit style slit lamp (SL-990) and a high-speed camera (X-PRI AOS Technologies AC) working at $500 \mathrm{fps}$ with a spatial resolution of $800 \times 560$ pixels. The left eye was illuminated through a slit 1-mm-wide at $45^{\circ}$ to the nasal part. The camera, which was situated to form $90^{\circ}$ with the light source, recorded the light diffused by the eye. The sequence was stored in the internal memory of the camera and later transferred to the computer through a fast ethernet connection.

The luminosity of the registered image with an exposure of $2 \mathrm{~ms}$ was very low. Modern slit lamps provide a setup for capturing images through the biomicroscope objective, but the optics of the system seriously limit the amount of light. To overcome this problem and get a sharp and luminous image, we placed the camera apart from the optical setup with an attached Navitar Zoom 7000 Macro Lens.

The images from the blinking sequences [Fig. 1(a)] were automatically processed off-line with our own software in MATLAB (Mathworks, Inc.). All the frames of each sequence were thresholded according to the background median value of the first slice [Fig. 1(b)]. This value was estimated from the upper left corner of the image. After binary thresholding, a morphological opening filter [7] removed isolated objects smaller than 50 pixels, thus leaving a clear profile of the anterior segment of the eye [Fig. 1(c)]. Finally, in order to clean residual salt and pepper noise, a two-dimensional (2D) median filtering was performed [Fig. 1(d)].

Processed images were arranged in a stack, thus forming a three-dimensional (3D) hypermatrix allowing 3D reslicing of the sequence and obtaining different time sections of the image (Fig. 2). The segment was taken perpendicular to the lower part of the cornea in order to prevent premature occlusion by the eyelid and to avoid 
the remaining noise in the center of the image. Selected sections are disposed as rows in a new image matrix, so that the temporal sequence can be directly observed, making clear the blinking dynamics. In the sequence, also called a kymogram, each image represents an axial section of the anterior segment of the eye.

Quantitative measuring of real displacements requires previous calibration of the images, i.e., obtaining the pixelto-millimeter ratio (PMR). Images from Fig. 1 correspond to an axial section of the anterior segment of the eye. Eye position may change between observers and so does the magnification. Thus, instead of using an absolute calibration for all sequences, we decided to calculate the PMR in each case. The central corneal thickness, measured with a Scheimpflug camera (Pentacam, Oculus Optigerate $\mathrm{GmbH}$ ), has been taken as a personal calibration distance and was assigned to the observed optical thickness in pixels. Thicknesses were measured by hand on five unprocessed frames randomly chosen for each sequence, thus obtaining a different PMR in each case. Because this measurement is affected by the corneal refractive index, the PMR has to be corrected in order to obtain real free-space distances. The average calibration distance so obtained was $42 \pm 9 \mathrm{pixel} / \mathrm{mm}$. The intraobserver dispersion was below 10\%, but the PMR for different observers ranged from 37 to $57 \mathrm{pixel} / \mathrm{mm}$, thus making the average calibration distance high. Further calculations were done with the proper sequence of PMR, thus reducing the dispersion of the results.

In Fig. 2, one can recognize two white regions corresponding to diffusion of light by the cornea (lower band) and the iris. The bands are interrupted by the crossing of eyelids during the blinking. The regions before and after this interruption provide information about eye retraction. We focus our attention on the lower border, which represents the first corneal surface. The method also allows a simultaneous measurement of the dynamics of the eyelid during blinking. The light reflected on the skin saturates the image so, as the eyelid closes or opens, the saturated area changes frame to frame. Thus, the variation in the intensity mean value of the image provides direct information about the eyelid position. The maximum intensity in a sequence was associated to the completely closed eye, while the minimum one was related to the maximum aperture of the eye. In Fig. 3, we depict the superposition of the eyelid displacement computed from the intensity mean value of each frame, with the eye retraction for one observer. All observers presented similar graphs. The position of the cornea at the beginning of the sequence was arbitrarily selected as the reference point. Small variations may lead to negative eye positions,

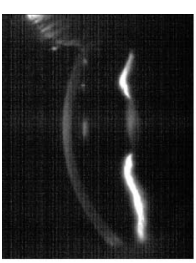

(a)

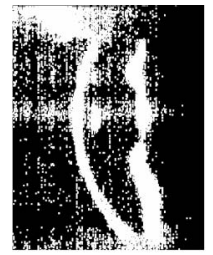

(b)

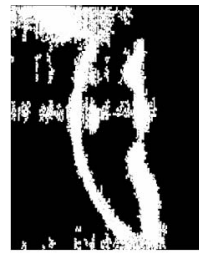

(c)

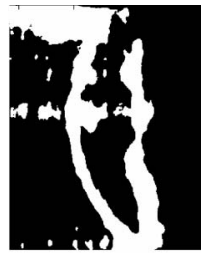

(d)
Fig. 1. Filtering process of the anterior segment image: (a) original image, (b) image after thresholding, (c) image after an opening filter, (d) final image after a 2D median filtering.

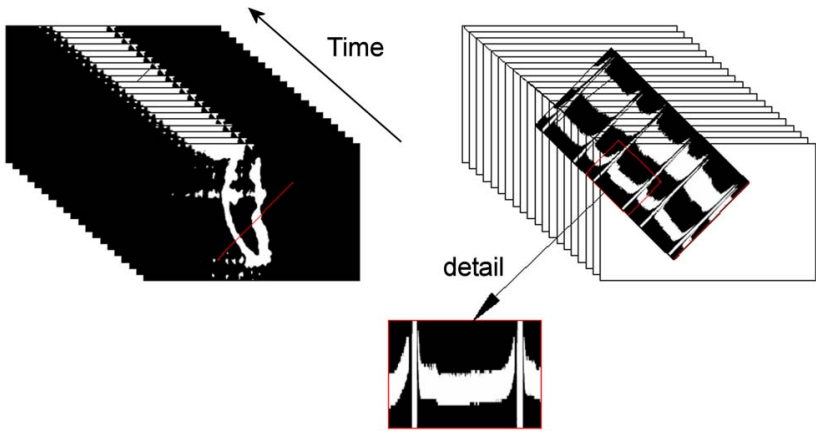

Fig. 2. (Color online) Schematic representation of the process followed to obtain the kymogram. The frames are stacked in a hypermatrix. A transversal plane is then extracted from the structure. The figure also shows a detail of the kymogram.

which are not real. The blinks can be recognized as a fast increase/decrease of the represented values. In the interblinking period, the eye is open and remains in a stable position. Suddenly, the eye starts a retraction up $1 \mathrm{~mm}$ as the eyelids close. When the eyelid opens again, the eye comes back to its previous position. The interruptions of the corneal line in the graphs show the moments when the eyelid crosses the scanning line, so corneal position is measured before and after these interruptions. References related to eye retraction during blinking establish the retraction between 0.44 [3] and 1-1.5 mm [8]. We obtained an average displacement of $0.8 \pm 0.2 \mathrm{~mm}$. Eye rotation toward the nose will produce an apparent retraction because the scanned segment changes. We have estimated this apparent displacement in $20 \mu \mathrm{m}$, in the limit of resolution of the method, so the observed effect can only be explained by an eye retraction. It is difficult to measure the blinking duration from corneal data, and we could not find any reference to validate our results. However, from eyelid motion, we measured blinking with an average duration of $0.307 \pm 0.106 \mathrm{~s}$, which is consistent with values found by VanderWerf et al. [9] and Nakamura et al. [10], who reported durations of 0.334 and $0.320 \mathrm{~s}$, respectively.

In Fig. 4, we show the velocity of the displacement of both eye and eyelid. The analysis was performed for all subjects, but a typical case is shown here. There is a coincidence in time between eye retraction and eyelid movement. It can be observed that the down-phase in the eye and eyelid starts simultaneously, while normal corneal position seems to be reached before the movement of the eyelid is finished. The synchronization

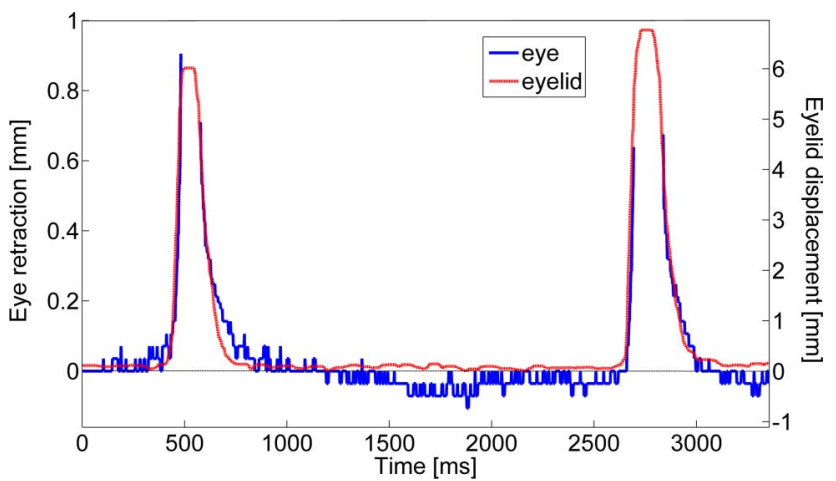

Fig. 3. (Color online) Eye retraction and eyelid position (calculated from image brightness) obtained for one typical subject. 


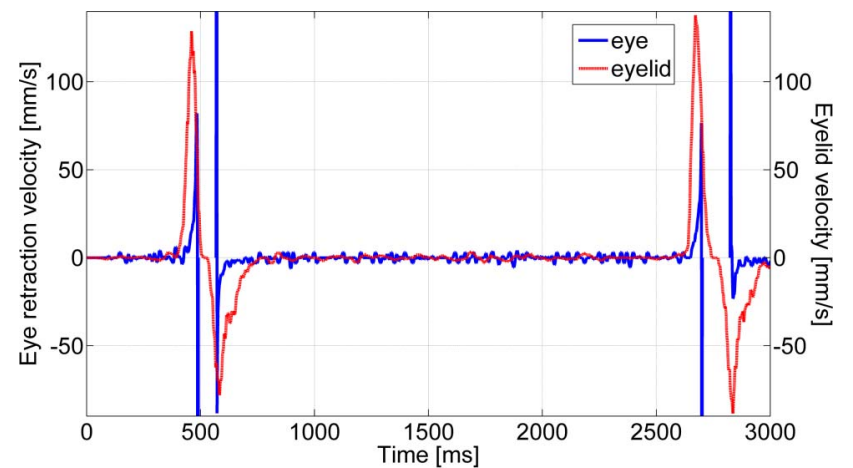

Fig. 4. (Color online) Velocity of eye retraction and eyelid movement computed for the subject in Fig. 3 .

between these movements is poorly described in the literature [2]. According to our results, both situations can be found, but in no case is the eyelid movement previous to the eye retraction.

The up and down phases can be clearly identified in the eyelid, together with the maximum velocity peaks and the different duration of both phases of blinking. The maximum velocities during closing and opening phases were $138 \pm 27$ and $96 \pm 18 \mathrm{~mm} / \mathrm{s}$, respectively. Other authors obtain similar values for these parameters: between 120 and $180 \mathrm{~mm} / \mathrm{s}$ for maximum closing speed, and between 45 and $82 \mathrm{~mm} / \mathrm{s}$ for opening speed, with dispersions of the order of $30 \%[5,10,11]$. Such high dispersions may be surprising; however, the dynamics of eye blinking depend on the sex, age, race, pathologies, environment, and even cultural and emotional factors [11], so intersubject comparison is difficult and only bulk values can be obtained.

The comparison of average and peak velocities of eye retraction with those of the eyelid shows that both are lower. We can also see that peak values are not reached in the same moment for eye and eyelid. It is not possible to establish the exact moment when the eye velocity reaches its maximum because it is occluded by the eyelid. Again, we are not able to validate these results, because no reference has been found about this issue. Physiological implications of such temporal displacements may be interesting, but this issue is outside the scope of this communication.

In summary, we have presented a noninvasive method for measuring eye retraction during blinking. The temporal and spatial accuracies are higher than existing noninvasive methods and are of the order of common invasive techniques. The method also allows simulta- neous evaluation of eyelid movement, thus permitting, for the first time, synchronized measurement of eyelid and eye activity. Obtained results and dispersions are comparable to those reported by other authors. We have also reported new results that are not described in the literature. Deep connection between ocular motion and neurophysiologic activity suggests that the analysis of synchronization of all movements in the blinking process can provide a better insight on brain activity.

The main drawback of our technique, when compared with invasive ones, is that the eyelid occludes the corneal image in some moments during the process, so the scanning segment is forced to be in the inferior part of the eye. Nevertheless, data losses can be minimized, and the improvement in the observer's comfort gives a more spontaneous blinking pattern to compensate for this limitation. Finally, we would like to emphasize that these results have been achieved through classical optical instrumentation and without the need of anesthetics, thus being within reach of many research labs.

This work has been supported by the Generalitat Valenciana project GV/2009/002. We also acknowledge Henryk Kasprzak for helpful discussions and comments.

\section{References}

1. B. J. Kirkwood, Insight 31, 15 (2006).

2. C. Evinger, M. D. Shaw, C. K. Peck, K. A. Manning, and R. Baker, J. Neurophysiol. 52, 323 (1984).

3. M. Collins, W. Smyth, J. Seawright, and S. Kelly, Clin. Exp. Optom. 75, 38 (1992).

4. H. Collejwin, in Vision Research: A Practical Guide to Laboratory Methods, R. H. S. Carpenter and J. G. Robson, eds. (Oxford U. Press, 1998), pp. 245-285.

5. S. H. Choi, K. S. Park, M. W. Sung, and K. H. Kim, Med. Biol. Eng. Comput. 41, 146 (2003).

6. M. Gora, K. Karnowski, M. Szkulmowski, B. J. Kaluzny, R. Huber, A. Kowalczyk, and M. Wojtkowski, Opt. Express 17, 14880 (2009).

7. J. Serra, Image Analysis and Mathematical Morphology (Academic, 1983).

8. O. Bergamin, S. Bizzarri, and D. Straumann, Invest. Ophthalmol. Visual Sci. 43, 3438 (2002).

9. F. VanderWerf, P. Brassinga, D. Reits, M. Aramideh, and B. O. de Visser, J. Neurophysiol. 89, 2784 (2003).

10. Y. Nakamura, J. Matsuda, K. Suzuki, H. Toyoda, N. Hakamata, T. Shimamoto, and S. Kiroshita, Nippon Ganka GakkaiZasshi 112, 1059 (2008).

11. C. Sforza, M. Rango, D. Galante, N. Bresolin, and V. F. Ferrario, Ophthal. Physiol. Opt. 28, 345 (2008). 